ARTICLE

DOI: $10.1038 / s 41467-017-00844-6$

OPEN

\title{
Direction-specific interaction forces underlying zinc oxide crystal growth by oriented attachment
}

\author{
X. Zhang ${ }^{1}$, Z. Shen', J. Liu ${ }^{2}$, S.N. Kerisit', M.E. Bowden², M.L. Sushko (1) ${ }^{1}$, J.J. De Yoreo ${ }^{1}$ \& K.M. Rosso ${ }^{1}$
}

Crystallization by particle attachment is impacting our understanding of natural mineralization processes and holds promise for novel materials design. When particles assemble in crystallographic alignment, expulsion of the intervening solvent and particle coalescence are enabled by near-perfect co-alignment via interparticle forces that remain poorly quantified. Here we report measurement and simulation of these nanoscale aligning forces for the $\mathrm{ZnO}$ (0001)- $\mathrm{ZnO}(000 \overline{1})$ system in aqueous solution. Dynamic force spectroscopy using nanoengineered single crystal probes reveals an attractive force with $60^{\circ}$ rotational periodicity. Calculated distance and orientation-dependent potentials of mean force show several attractive free energy wells distinguished by numbers of intervening water layers, which reach a minimum when aligned. The calculated activation energy to separate the attractively bound solvated interfaces perfectly reproduces the measured $60^{\circ}$ periodicity, revealing the key role of intervening water structuring as a basis to generate the interparticle torque that completes alignment and enables coalescence.

\footnotetext{
${ }^{1}$ Physical and Computational Sciences Directorate, Pacific Northwest National Laboratory, Richland 99352 WA, USA. ${ }^{2}$ Environmental Molecular Sciences Laboratory, Pacific Northwest National Laboratory, Richland 99352 WA, USA. X. Zhang and Z. Shen contributed equally to this work. Correspondence and requests for materials should be addressed to K.M.R. (email: kevin.rosso@pnnl.gov)
} 
C rystallization by particle attachment (CPA) in solution is a widespread phenomenon in geochemical, biomineral, and synthetic material systems ${ }^{1-4}$. Unlike classical crystal growth via ion addition, CPA is facilitated by aggregation of particles, typically nanosized, that coalesce, recrystallize, or assemble into larger structures ${ }^{1,4-6}$. A complete picture of CPA, of course, also includes classical monomer-by-monomer dissolution, precipitation, and ripening during the particle motion, collision, and aggregation ${ }^{1}$. Oriented attachment (also known as "oriented aggregation") is a special case in which aggregating nanocrystals self-assemble into extended lattices through preferential attachment on specific crystal faces. Since its discovery in natural systems $s^{2,3}$ there has been growing interest in exploiting oriented attachment to construct hierarchically structured crystalline materials. Examples include metals such as $\mathrm{Au}^{7}$ and $\mathrm{Ag}^{8}$, alloys such as $\mathrm{ZnTe}^{9}, \mathrm{Pt}-\mathrm{Ni}, \mathrm{Pt}-\mathrm{Cu}$, and $\mathrm{Pt}-\mathrm{Fe}^{10}, 11$, metal oxides such as $\mathrm{ZnO}^{12}, 13, \mathrm{TiO}_{2}{ }^{3}, \mathrm{MnO}^{14}$, and $\alpha-\mathrm{Fe}_{2} \mathrm{O}_{3}{ }^{15}$, metal sulfides such as $\mathrm{PbS}^{16}, \mathrm{ZnS}^{17}, \mathrm{Ag}_{2} \mathrm{~S}^{18}$, and $\mathrm{CdS}^{18,}{ }^{19}$, and metal selenides such as $\mathrm{PbSe}^{20}, \mathrm{CdSe}^{19}, \mathrm{NiSe}_{2}{ }^{21}$. Furthermore, surfactants have been widely used in experiments to facilitate oriented particle assembly via preferential adsorption on crystal surfaces. The proposed roles of surfactants in aligning nanoparticles and promoting oriented attachment include surfactant interaction-related driving forces for self-assembly or mesocrystal formation ${ }^{22-24}$, stabilization of kinetically metastable intermediate nanoparticles, and control of coalescence crystal face or the overall shape of the mesocrystal $^{20,24}$.

Face selectivity that enables oriented attachment conceptually arises from interparticle forces that are both sensitive to mutual crystallographic orientation and strong enough to rotate approaching particles into lattice alignment ${ }^{1,3}$. However, so far these forces have been more often inferred than measured. For example, recent developments in liquid cell transmission electron microscopy (TEM) imaging technologies have provided opportunities to investigate crystal growth by oriented attachment in situ in real time ${ }^{11,25}$. Such a study of the iron oxyhydroxide ferrihydrite in aqueous solution showed that, during particle encounters, a nanoscale solvent-separated state was maintained in which nominally random mutual rotational motions were overtaken by stronger directional forces when close to crystallographic alignment, enabling successful oriented attachment and coalescence $^{25}$. For dipolar nanoparticles such as $\mathrm{Pt}_{3} \mathrm{Fe}$, a strongly anisotropic assembly pattern was observed in which chains were formed by end-to-end attachment conceptually consistent with interparticle dipole alignment ${ }^{11}$. Clearly, assembly is governed by forces capable of creating interparticle torques strong enough to enable alignment while particles are still solvent separated, but to date these forces remain poorly quantified.

It is well-known that the interfacial forces between two flat solid surfaces in solution strongly depend on the atomic structures of the surfaces and the intervening solvent molecules, especially at short range. For example, the interaction between two macroscopic mica basal surfaces in aqueous solution measured using the surface force apparatus (SFA) showed oscillatory repulsive hydration forces consistent with structured intervening water layers, and adhesive force maxima at twist angles of $0^{\circ} \pm$ $60^{\circ}, \pm 120^{\circ}$, and $180^{\circ}$, consistent with azimuthal alignment of the mica-mica lattices ${ }^{26}$. However, extending these kinds of studies with control of mutual crystallographic orientation and solution conditions into the nanoscale domain of oriented attachment systems is experimentally challenging. One study reported atomic force microscopy(AFM)-based force measurements between a micron-sized oriented gypsum single crystal and opposing substrate $^{27}$. But to understand the forces enabling oriented attachment, measurements of the direction-specific interaction forces on nanocrystals in situ is an essential step. The recently developed
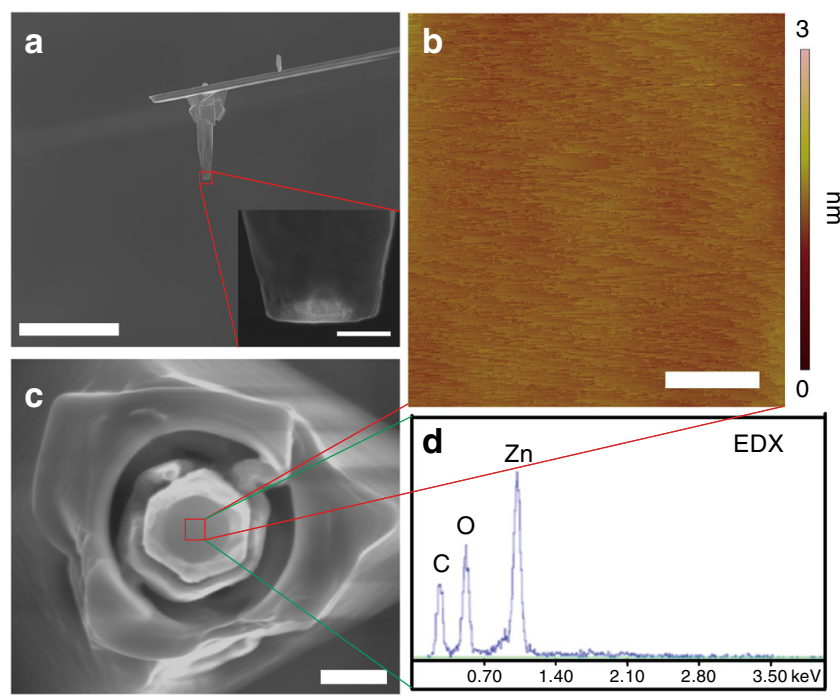

Fig. 1 SEM and AFM images of a $\mathrm{ZnO}(0001)$ AFM tip. a Side view (scale bar $10 \mu \mathrm{m}$ ), the inset (scale bar $100 \mathrm{~nm}$ ) is a high magnification image to show surface flatness; b Top view (scale bar $500 \mathrm{~nm}$ ); c an AFM image of the $\mathrm{ZnO}$ tip surface, collected by reverse imaging using a whisker substrate (scale bar $25 \mathrm{~nm}$ ); and $\mathbf{d}$ an EDX spectrum of the $\mathrm{ZnO}$ tip surface

in situ environmental TEM-AFM technique also allows measurements of direction-specific interaction forces between nanocrystals but is limited to high-vacuum or low-water vapor conditions ${ }^{28}$.

Here we report AFM-based measurements of the forces on an oriented face-specific single nanocrystal probe as it interacts with its symmetrically related counterpart crystal face in aqueous solution using dynamic force spectroscopy (DFS) (Supplementary Fig. 1). By controlling the azimuthal alignment, the measurements test the dependence of the interaction on crystal symmetry, a fundamental prerequisite of oriented attachment. The novelty of the experimental aspect of our study is precision control of the tip crystallographic face expression, area, and orientation at the nanoscale. Also, by measuring the jump-from-contact force as a function of tip retraction rate, the rupture force at equilibrium and the corresponding adhesion-free energy as a function of azimuthal alignment is directly obtained ${ }^{29}$. We compare the measured orientation dependence of the adhesion-free energy to predictions of the potential of mean force (PMF) from large-scale molecular dynamics (MD) simulations of the solvated interparticle gap. From the combined results we demonstrate that the sensitivity to crystallographic alignment between nanocrystals at close range arises, at least in part, from the self-organization of intervening water molecules in response to the structures and alignment of the opposing surfaces. Furthermore, we show that alignment creates an attractive energy minimum with fewer intervening water layers between the surfaces, enabling closer approach. The findings show that the solvent structure in the interparticle region creates an energy barrier that helps maintain the solvent separated state.

$\mathrm{ZnO}$ is an important material in semiconductor and microelectric industries ${ }^{30-32}$. It has been used to fabricate nanogenerators $^{33}$, solar cells ${ }^{34,35}$ LEDs $^{36}$, and photo sensors ${ }^{37}$, and as a photocatalyst to degrade organic pollutants ${ }^{38}$. Both theoretical calculations and experimental data have indicated the $\mathrm{ZnO}$ nanocrystals oriented aggregate through the $(001) /(002)$ surface to form the rod-like single crystals ${ }^{12,13,39}$. Thus our study focuses on $\mathrm{Zn}$-terminated $\mathrm{ZnO}(0001)$ interaction with its partner O-terminated $\mathrm{ZnO}(000 \overline{1})$ face as a model oriented attachment 
system. $\mathrm{ZnO}$ has a hexagonal wurtzite-type structure $\left(\mathrm{P}_{3} \mathrm{mc}\right)$, which is composed of alternating planes of tetrahedrally coordinated $\mathrm{O}^{2-}$ and $\mathrm{Zn}^{2+}$ ions, stacked along the $c$-axis in an $\mathrm{ABAB}$ pattern (hexagonal close packing) (Supplementary Fig. 2$)^{30}$. This stacking of oppositely charged ions produces a net dipole moment normal to the basal (0001) plane $\mathrm{e}^{31}$. An opposing pair of Tasker type III polar bulk truncations is thus obtained, which reconstruct to form stable (0001) and $(000 \overline{1})$ terminations.

\section{Results}

Force measurement method. For our force measurements, a $(000 \overline{1})$ terminated bulk $\mathrm{ZnO}$ crystal was used as a substrate, and (0001) terminated probes were nanoengineered onto AFM cantilevers (Methods section, Supplementary Note 1, Supplementary Figs. 3, 4 and 5). The resulting $\mathrm{ZnO}(0001)$ AFM tips (Fig. 1) showed $\sim 0.5 \mathrm{~nm}$ roughness arising from stepped atomically flat terraces as determined by high resolution reverse AFM imaging, and exposed clean stoichiometric surfaces as determined by crosssectional high resolution scanning electron microscope (SEM) and energy-dispersive X-ray spectroscopy (EDX). Measurements were performed in flowing $0.2 \mathrm{mM} \mathrm{Zn}\left(\mathrm{NO}_{3}\right)_{2}$ solution (Methods section, Supplementary Note 2, and Supplementary Fig. 6). Given the hexagonal symmetry of $\mathrm{ZnO}$ along its $c$ axis, the azimuthal alignment was varied from $0^{\circ}$ to $120^{\circ}$ during the force measurements.

Measured $\mathrm{ZnO}(0001)-\mathrm{ZnO}(000 \overline{1})$ interaction force. DFS was used to investigate $\mathrm{ZnO}(0001)-\mathrm{ZnO}(000 \overline{1})$ interaction as a function of azimuthal orientation in saturated aqueous solution ${ }^{29}$. Figure 2a shows a typical force versus distance curve, and the force measurement process is shown in Supplementary Movie 1. Point 1 to 2 presents the approach process, in which the $\mathrm{ZnO}$ (0001) AFM tip was pushed to contact the $\mathrm{ZnO}(000 \overline{1})$ substrate via a fixed approaching rate. During tip approach a jump-tocontact event was always evident (point $2-3$ in Fig. 2a), indicating the presence of an attractive force gradient exceeding the cantilever stiffness $\left(\sim 0.2 \mathrm{nN} \mathrm{nm}^{-1}\right)$. Then the tip was pushed further to contact the substrate from point 3-4 until the contact ramp. A maximum loading force of $5 \mathrm{nN}$ was applied with a dwell time of $2 \mathrm{~s}$ to stabilize the interaction. Tip retraction was performed (point 4-5) via different loading rates and was always terminated by a larger jump-from-contact event (point 5-6) consistent with strong attractive interaction. To estimate the equilibrium rupture force $\left(f_{e q}\right)$, sets of force curves at five different pulling rates were collected and $f_{e q}$ at each azimuthal orientation was obtained by fitting the data to the multiple bond model of Friddle et al. ${ }^{29}$.

Figure $2 \mathrm{~b}$ shows the measured jump-from-contact forces at different azimuthal orientations from $0^{\circ}$ to $120^{\circ}$ for a selected tip area ( $381 \mathrm{~nm}$ diameter). Because the crystallographic orientation of the $a$ and $b$ axes of the $\mathrm{ZnO}$ substrate was pre-determined from single crystal X-ray diffraction (Supplementary Fig. 7), and because the same was known for the tip based on its $\mathrm{ZnO}$ nanowire morphology, measurements could be performed based on absolute azimuthal orientations (Supplementary Note 3). The force decreases with azimuthal orientation progressing from $0^{\circ}$ to $30^{\circ}$ for each pulling rate, then increases with azimuthal orientation progressing from $30^{\circ}$ to $60^{\circ}$. Another force minimum and maximum are obtained upon progressing from $90^{\circ}$ to $120^{\circ}$. For comparison, the interaction force between the $\mathrm{ZnO}(0001)$ tip and a flat silicon wafer substrate bearing the usual amorphous silicon oxide passivation layer was also measured (Fig. 2b); in this case the interaction is rather weak and no periodicity is observed, as expected. Figure $2 \mathrm{c}$ shows the fitted curves for azimuthal orientations from $0^{\circ}$ to $60^{\circ}$. All resulting equilibrium interaction forces are plotted in Fig. $2 \mathrm{~d}$ and clearly display a cyclic nature with a repeat period of $60^{\circ}$ and force maxima at $0^{\circ}, 60^{\circ}$, and $120^{\circ}$. To approximate the average maximum and minimum rupture force, the data were fit using a simple sinusoidal waveform (Methods section). A maximum rupture force of $79.78 \mathrm{nN}$ was obtained when the azimuthal orientation is $0^{\circ}, 60^{\circ}$, and $120^{\circ}$ and a minimum rupture force of $47.30 \mathrm{nN}$ was obtained when the azimuthal orientation is $30^{\circ}$ and $90^{\circ}$. The maximum and minimum interfacial forces thus occur in a repeating manner that is twice the bulk crystallographic periodicity of the $\mathrm{ZnO}$ wurtzite crystal structure along the $c$ axis.

The effect of contact area on the interaction force was investigated using tips fabricated with systematically varying diameters ranging from $381 \mathrm{~nm}$ down to $172 \mathrm{~nm}$ (Fig. 3). In all cases, the derived equilibrium interaction force exhibits cyclical dependence on azimuth with a period of $60^{\circ}$ (Supplementary Fig. 8). The determined maximum and minimum rupture forces for seven different contact areas are plotted in Fig. 3a, which show a linear dependence on contact area. When normalized to the tip surface area, the maximum and minimum rupture forces are approximately constant, showing a difference of around $5 \times 10^{5}$ $\mathrm{N} \mathrm{m}^{-2}$ (Fig. 3b). This result supports our conclusion that face-toface contact between the $\mathrm{ZnO}(0001)$ AFM tips and the $\mathrm{ZnO}$ $(000 \overline{1})$ substrate was generally achieved during the force measurements.

The energy required to overcome the stiffness of the AFM cantilever when it reaches $f_{\text {eq }}$ is given by ${ }^{40}$ :

$$
E=\frac{f_{\mathrm{eq}}^{2}}{2 k_{\mathrm{c}}}
$$

where $k_{c}$ is the cantilever spring constant. The value $E$ corresponds to the free energy difference between the bound and unbound states, and is thus the adhesion-free energy. The value $E / 2$ is the interfacial free energy per $\mathrm{ZnO}$ surface, averaged for the two different terminations. The resulting adhesion-free energies show a linear dependence on the tip contact area (Fig. 3c), and after area normalization the maximum and minimum adhesion-free energy are obtained (Fig. 3d). The maximum values are more than an order of magnitude lower than the cleavage energy of $\mathrm{ZnO}$ along the $c$ axis $\left(\sim 4.0 \mathrm{~J} \mathrm{~m}^{-2}\right)^{41}$, which is the theoretical upper limit, consistent with the likelihood that some of the waters of hydration remain in the interfacial region during contact, as discussed below. Corresponding interfacial free energies $\left(\sim 0.015-0.053 \mathrm{~J} \mathrm{~m}^{-2}\right)$ are also substantially lower than measured hydrated surface enthalpies of $\mathrm{ZnO}$ nanocrystals $\left(\sim 1.3 \mathrm{~J} \mathrm{~m}^{-2}\right)^{42}$, consistent with the clear difference in our measurements that hydration water is confined between the two $\mathrm{ZnO}$ surfaces. The difference between the maximum and minimum adhesion-free energy was regularly $\sim 0.076 \mathrm{~J} \mathrm{~m}^{-2}$.

Molecular simulation of $\mathrm{ZnO}(0001)-\mathrm{ZnO}(000 \overline{1})$ interactions. To have a better understanding of the origin of the observed angular dependence of the interaction, we carried out PMF calculations for $\mathrm{ZnO}(0001)-\mathrm{ZnO}(000 \overline{1})$ solvated in water using empirical atomistic force fields and MD simulations (Methods section). In the simulation system the (0001) termination of a hexagonal $\mathrm{ZnO}$ nanoparticle faces the $(000 \overline{1})$ termination of a 2-D $\mathrm{ZnO}$ slab, surrounded by water molecules. In the simulations, the orientation and position of the substrate are fixed while the particle is placed in different azimuthal orientations with respect to the substrate $\left(0-120^{\circ}\right.$ with an interval of $\left.15^{\circ}\right)$ and the distance dependence of the PMF is computed at each orientation.

The resulting PMF curves at all azimuthal angles share a common feature. That is, there are three free energy wells at $\sim 5.8$, $\sim 7.5$, and $\sim 9.7 \AA$, respectively (Fig. $4 \mathrm{a}$ ). The first energy well corresponds to a configuration where there are two water layers 
a

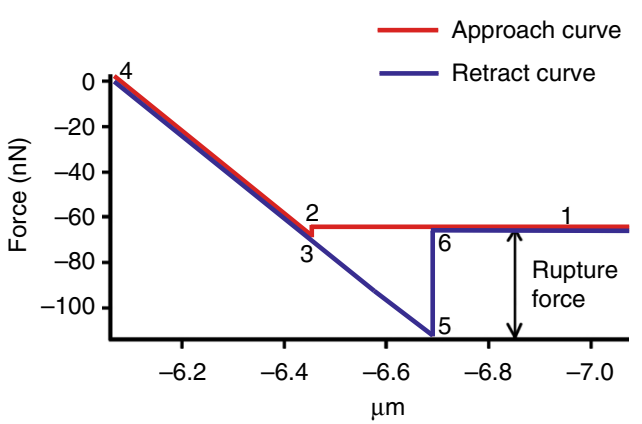

c

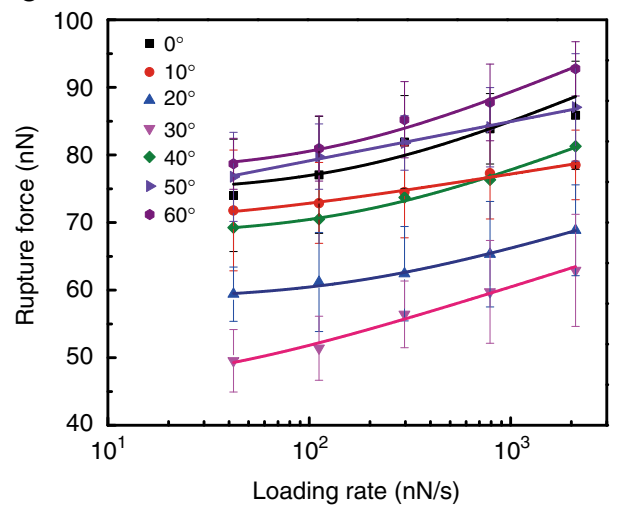

b

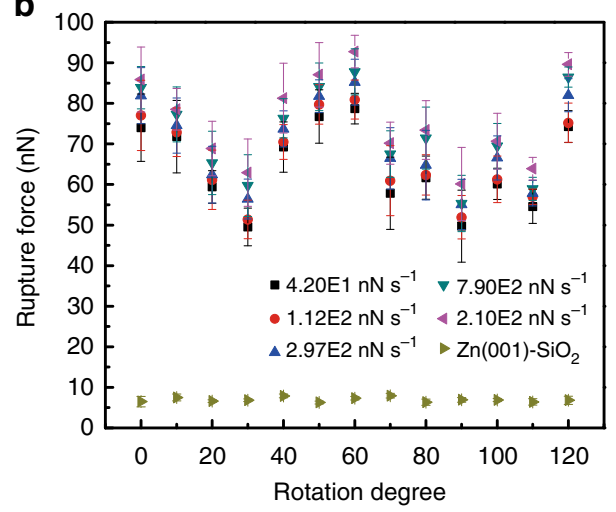

d

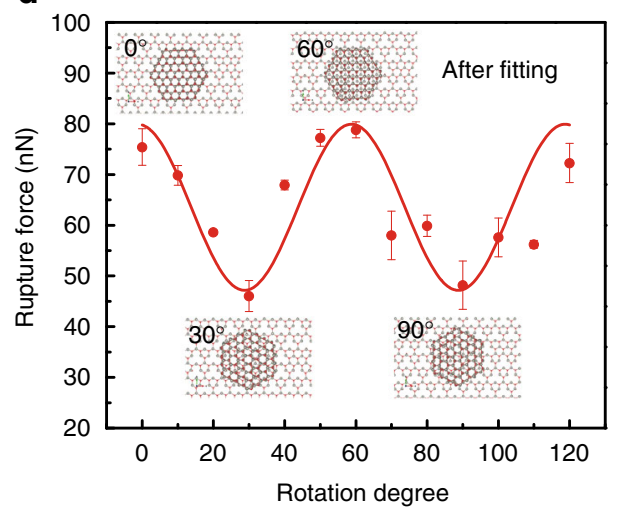

Fig. 2 Face-specific interaction force measurements. a Representative force curves for $\mathrm{ZnO}(0001)-\mathrm{ZnO}(000 \overline{1})$ interaction in saturated aqueous solution. Point 1-2 presents the approach process until the jump-to-contact happened (point 2-3); then further push the tip to contact the substrate from point 3-4; after a short time to stabilize the interaction, tip retraction was performed (point 4-5) until a jump-from-contact happened (point 5-6). b Direction-specific interaction forces at different azimuthal orientation for $\mathrm{ZnO}(0001)-\mathrm{ZnO}(000 \overline{1})$ and $\mathrm{ZnO}(0001)-\mathrm{SiO}_{2} / \mathrm{Si}$ and different loading rates for $\mathrm{ZnO}(0001)-\mathrm{ZnO}$ $(000 \overline{1})$. c Data fitting for the different loading rates at each azimuthal orientation from $0^{\circ}$ to $60^{\circ}$ to obtain the equilibrium rupture force. Error bars in $\mathbf{b}$ and $\mathbf{c}$ represent the s.e. $(n=25)$. d Rupture forces at different azimuthal orientation after fitting using the multiple bond model developed by Friddle et al. ${ }^{29}$; Error bars derived from the fitting of the multiple bond model. Inset illustrates corresponding structure relationships between the tip and substrate crystals at selected azimuthal orientations $\left(0^{\circ}, 30^{\circ}, 60^{\circ}\right.$, and $\left.90^{\circ}\right)$

in-between the two opposing surfaces. The first layer water molecules are located above vacancies created to remove the surface dipole of the $\mathrm{ZnO}(000 \overline{1})$ slab. In the second layer, the water molecules form a hydrogen-bonded network with both the first water layer and atoms on the $\mathrm{ZnO}(0001)$ surface of the particle. Correspondingly, three water layers are found to exist between the two surfaces in the configuration at the second well, and four water layers are associated with the third well.

Based on the number and character of the wells as a function of azimuthal orientation, the PMF curves can be divided into three groups (one exemplary force curve and PMF curve of each group are shown in Supplementary Fig. 9). The first group is composed of the PMFs at $0^{\circ}$ (or equivalently $120^{\circ}$ ) and $60^{\circ}$, in which the energies at the first well are strongly negative $(-0.36$ to $-0.40 \mathrm{eV})$ and much more attractive in character than at any other wells found in the system. In the second $\left(15^{\circ}, 45^{\circ}, 75^{\circ}\right.$, and $\left.105^{\circ}\right)$ and third $\left(30^{\circ}\right.$ and $\left.90^{\circ}\right)$ groups, the energies at distances corresponding to this first well are positive but distinct in terms of how positive, with the latter being more positive than the former. The global minimum for both groups 2 and 3 is at a distance corresponding to the second energy well of the first group.

For comparison with the orientation dependence of the adhesion-free energies determined by DFS, we computed corresponding values from the MD simulations, taken as the difference between an energy minimum and zero energy at a far separation for each azimuthal angle, normalized to interfacial area
(Fig. 4b). If we consider the second well, where three layers of intervening water persist at all azimuthal angles (Fig. 4b, red line), a periodicity of $60^{\circ}$ is evident, identical to that observed from the force measurements. This periodicity, again occurring as twice the bulk crystallographic periodicity, can now be clearly identified as corresponding to the period of dominant alignment of opposing surface cation and anion sub-lattices across the solvated interface. At $0^{\circ}$ and $120^{\circ}$ the two lattices are in symmetry equivalent bulk crystallographic alignment along the $c$-axis. At $60^{\circ}$, one lattice has a translation of $1 / 3\langle 01 \overline{1} 0\rangle$ with respect to the other, and thus the interface resembles the very common type-I stacking fault with a stacking sequence of (...AaBbAaCcAaCc...) in the wurtzite structure $^{43}$. This type of stacking fault has been shown to have a very low formation energy ${ }^{44}$. As one major finding from the simulations, it appears that this low-energy configuration manifests even across the solvent-filled gap.

Calculated maximum and minimum adhesion-free energies as a function of azimuth generally compare remarkably well with those determined by DFS. For the second well they are lower by a factor of approximately 10 . However, if the global minimum well at each angle is considered, which includes the first well for crystallographically aligned angles (Fig. 4b, black line), the agreement improves to a factor of 5 . The adhesion-free energy is substantially larger when the two surfaces are aligned, where only two water layers are found to persist between them. In this case, the adhesion-free energy difference per contact area between 

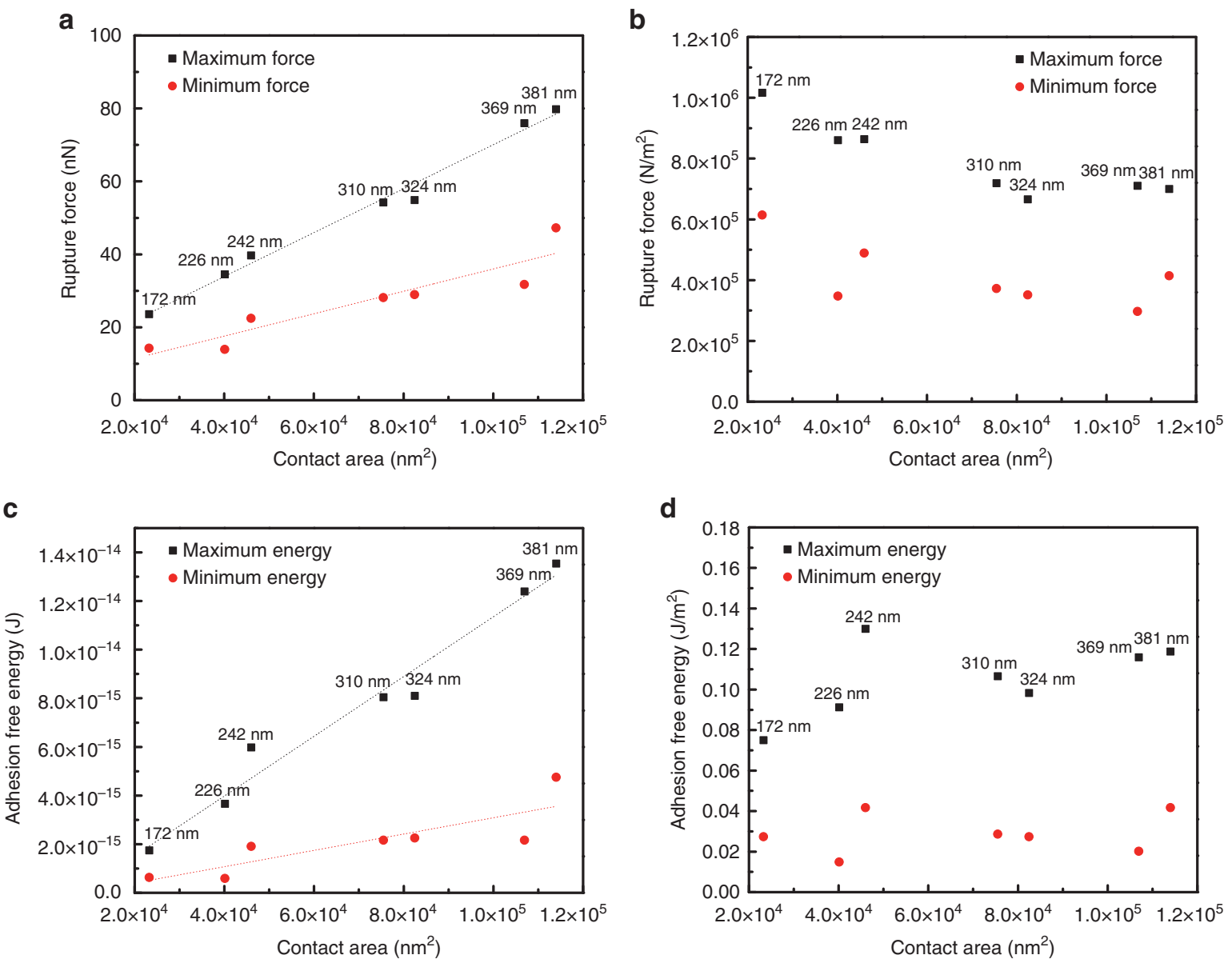

Fig. 3 Maximum and minimum rupture forces and adhesion-free energies of $\mathrm{ZnO}(0001)-\mathrm{ZnO}(000 \overline{1})$. a Force versus tip contact area; $\mathbf{b}$ force normalized to tip contact area; $\mathbf{c}$ the corresponding adhesion-free energy versus contact area; $\mathbf{d}$ adhesion-free energy normalized to tip contact area. Tip diameters are noted

$60^{\circ}$ or $120^{\circ}$ and $30^{\circ}$ or $90^{\circ}\left(\sim-0.015 \mathrm{~J} \mathrm{~m}^{-2}\right)$, as well as the relative ratio between these two values $(\sim 4.5: 1)$, show the best correspondence with the DFS data. The analysis strongly suggests that in the measurements, the deepest well for each angle is always achieved; the nanocrystal tip reached the first well when aligned at $60^{\circ}$ or $120^{\circ}$, and the second well at the rest of the angles. The $60^{\circ}$ periodicity continues to hold true regardless of which well is considered, and the overall correspondence between the experiments and simulation for the azimuthal dependence of the adhesion-free energy is striking.

Analysis of the PMF calculations can be extended to consider the energy barriers that correspond to moving the particle out of its global minimum to larger separations at each azimuthal angle (Fig. 4c). The azimuthal dependence of this detachment free energy barrier also shows a periodicity of $60^{\circ}$, with maximum values corresponding to aligned conditions and minimum values at misaligned conditions. With respect to an oriented aggregation process, these barriers suggest it is easier for nanoparticles attracted in a solvent-separated state to detach from each other when misaligned compared to when they are aligned, consistent with a higher probability of interparticle rotation when misaligned, maintaining the search for perfect lattice alignment. The inverse analysis of barriers for moving the particle from the second to the first well is somewhat precluded by the lack of a first minimum for misaligned angles (Fig. 4a), but by inspection of the PMFs these barriers would clearly be lowest for aligned conditions, consistent with a higher probability of attaining shorter interparticle separations when alignment is achieved.
To better understand the origin of this free energy landscape, we also performed PMF calculations in vacuum to compute solvent-independent contributions to particle-particle (P-P) interaction free energies at $90^{\circ}, 105^{\circ}$, and $120^{\circ}$, each representative of its own group (Fig. 4d). At all three angles, the PMF curves decrease monotonically with decreasing separation within the same distance range as that which we explored in the solution calculations. At $\sim 5.8 \AA$, the energy at $90^{\circ}$ is very close to that at $105^{\circ}(\sim 0.01 \mathrm{eV})$, and thus both are about $0.05 \sim 0.06 \mathrm{eV}$ higher than that at $120^{\circ}$. These small differences are not enough to account for the much larger difference in energy at the first energy well between group 1 and the other two groups and neither for the difference between group 2 and group 3 .

The water-mediated contribution to the free energy was obtained (as shown in the inset of Fig. 4d) as a difference between the total PMF in solution and the PMF in vacuum containing only P-P interactions. From the analysis of water contribution alone it immediately becomes clear that the watermediated forces play a decisive role in determining the shape of free energy curves as well as the angular dependence of interfacial interactions in our simulation system. The free energy oscillations with distance show a period of about the diameter of a water molecule, mimicking the well-known oscillatory solvation force that can occur between two solid surfaces at small separations $(<\text { a couple of } \mathrm{nm})^{45}$. On the other hand, the difference in watermediated forces, similar to the repulsive hydration force between two hydrophilic surfaces ${ }^{45-47}$, at different angles might be the origin of the orientation dependence of the interactions that emerge from our simulations, and by similarity the measured 
a

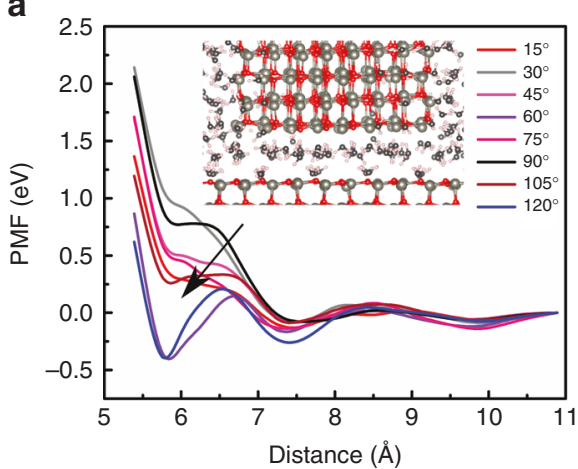

C

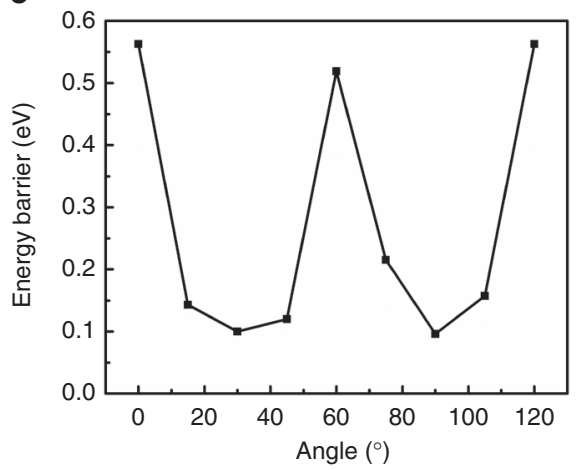

b

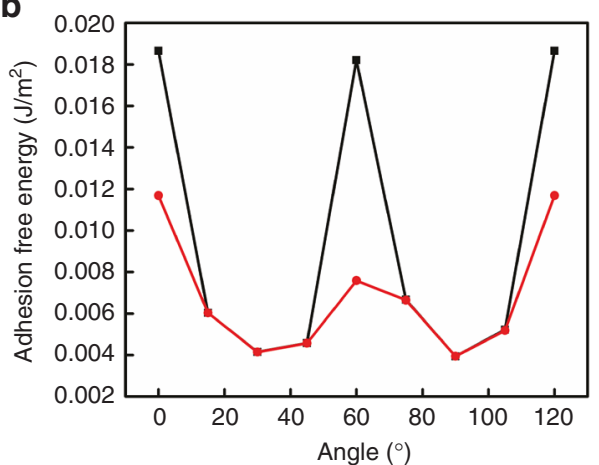

d

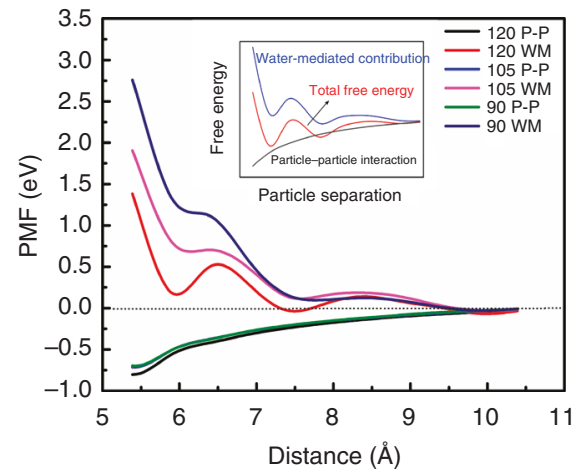

Fig. 4 Calculated PMF curves and energy barriers for $\mathrm{ZnO}(0001)-\mathrm{ZnO}(000 \overline{1})$ interactions at different azimuthal angles. a PMF curves at different azimuthal angles. An exemplary configuration at the first energy well is also shown. $\mathbf{b}$ Adhesion-free energies between $\mathrm{ZnO}$ particle and the substrate by using global minima (black filled circles) and second minima (red filled circles), at different angles. For better comparison with the adhesion-free energies estimated from experiments as shown in Fig. $3 \mathrm{~d}$, the free energies obtained from a are normalized to the surface area and the unit of $\mathrm{J} \mathrm{m}^{-2}$ is used. $\mathbf{c}$ Energy barriers for moving the particle out of the lowest energy minima at different angles. $\mathbf{d}$ Comparison of contributions to the total PMF from particle-particle (P-P) interactions and from the water-mediated forces at $90^{\circ}, 105^{\circ}$, and $120^{\circ}$. The total PMF and the P-P interaction contribution are calculated from MD simulations, and the water contribution is obtained as a difference between the previous two terms as shown in the inset

rupture force. The theory of hydration repulsion remains poorly understood due to its complex involvement of water-water interaction, water-surface interaction, and entropic contributions ${ }^{46}$. Our analysis of hydrogen bonds in-between the two $\mathrm{ZnO}$ surfaces shows (Supplementary Note 4) no obvious angular dependence on the number of hydrogen bonds and the hydrogen bond length (Supplementary Fig. 10). But we find a similar cyclic change with a period of $60^{\circ}$ in the number of $\mathrm{Zn}$ $\mathrm{O}_{\text {water }}$ bonds in the space between the two $\mathrm{ZnO}$ surfaces (Supplementary Fig. 11).

From this study, we find that classical MD/PMF calculations reproduce with striking similarity the same period of angular dependence of $\mathrm{ZnO}-\mathrm{ZnO}$ interfacial interaction as that observed in the rupture forces measured in solution. The complete correspondence reveals the prominent role of water structuring for "communicating" azimuthal alignment between like surfaces across the inter-surface, or in the case of oriented attachment inter-particle, solvent gap. Water structuring also creates attachment barriers that preferentially maintain the solvent-separated state during misalignment. The observed angular dependence of adhesion therefore arises less from P-P interactions and more from water-mediated forces that oscillate with mutual crystallographic alignment. The findings indicate that direction-specific interaction forces can create torque to align $\mathrm{ZnO}$ nanocrystals at close separations to induce the oriented attachment, which can explain the ex situ TEM observations that $\mathrm{ZnO}$ nanoparticles can aggregate via oriented attachment to form rod-like single crystals. The findings also can help to explain the oriented attachment behaviors of other material systems and understand the self- assembly of nanocrystals, Schiller layer formation, and even the grain boundary construction in polycrystals.

\section{Methods}

Force measurement. The studies were performed using an Asylum MFP-3D AFM. The experimental setup is shown in Supplementary Fig. 1. Atomically flat and oriented face-specific single crystals to be used as $\mathrm{ZnO}$ substrates were adhered to a flow chamber equipped with a rotation stage using Crystalbond ${ }^{\mathrm{im}} 509$ Mounting Adhesive (Structure Probe, Inc.). Another atomically flat and oriented face-specific single nanocrystal was loaded onto an AFM cantilever, using nanofabrication methods described in the following Methods section. The lattice orientation of the $(000 \overline{1}) \mathrm{ZnO}$ substrates (i.e., the $<10 \overline{1} 0>$ directions within the surface plane) was determined using $x$-ray diffraction and the lattice orientation of the (0001) ZnO AFM tip was determined using SEM (Supplementary Note 2). The azimuthal angles were adjusted by rotating the $(000 \overline{1}) \mathrm{ZnO}$ substrates in the fluid cell. The stability of the (0001) ZnO AFM tip was investigated in detail via using ex situ SEM (Supplementary Note 1) and the surface stability of the $\mathrm{ZnO}(000 \overline{1})$ bulk substrate was detected using in situ liquid cell AFM (Supplementary Movie 2).

Nanoscale ZnO(0001) AFM tip fabrication. Fabricating oriented face-specific $\mathrm{ZnO}(0001)$ nanosized AFM tips with atomically flat clean surfaces is a challenge. Beyond the difficulty of achieving these characteristics, the (0001) tip face must also be co-planar with the substrate surface throughout force measurements and stage rotation in order to maintain a known area of interaction. Among several fabrication methods the most tried is by glue-mounting single crystals using macroscopic techniques, which due to the difficulties of manipulation and orientation control is usually limited to micron-sized particles ${ }^{48}$. Another method is to grow materials on original AFM tips using physical vapor deposition (PVD), chemical vapor deposition (CVD), ion beam sputter (IBS) deposition, or electrodeposition ${ }^{49}$, but this invariably leads to polycrystalline materials. The method used in the present study is microscopic manipulation and mounting of oriented single $\mathrm{ZnO}$ nanowires (NWs) onto AFM cantilevers that are then downsized further to a controlled nanoscale surface area by focused ion beam (FIB) milling ${ }^{50}$. To avoid 
contamination of the tip surface by ion impact ${ }^{51}$, special care was required (Supplementary Fig. 4).

$\mathrm{ZnO} \mathrm{NW}$ arrays expressing hexagonal prismatic surfaces were synthesized on gold substrates via a hydrothermal method (Supplementary Fig. $4 \mathrm{a})^{52}$. The method produced NWs with the exposed upper wire surface as the Zn-terminated (0001) face $^{53}$. Individual $\mathrm{ZnO}$ NWs were nanomanipulated onto AFM cantilevers (Omniprobe, Oxford Instruments) inside of a SEM for real-time observation (Helios NanoLab 600i, FEI, Hillsboro, OR). For the specific AFM used for the force measurements, the cantilever was mounted with an $11^{\circ}$ tilt from the substrate, so the AFM tips onto which the $\mathrm{ZnO}$ NWs were mounted were first pre-milled to compensate for this tilt angle (Supplementary Fig. 5). To securely hold the $\mathrm{ZnO}$ NWs on the cantilever, a hole was then drilled into the tip, orthogonal to its tilted milled surface (Supplementary Fig. $4 \mathrm{~b}$ ). To transfer a $\mathrm{ZnO}$ NW onto the cantilever, the Omniprobe was driven close to a selected $\mathrm{ZnO} \mathrm{NW}$ and then a rectangular patch of platinum $(\mathrm{Pt})(50 \mathrm{~nm}$ thick) was deposited on its side surface by ionbeam-assisted deposition (Supplementary Fig. 4a), which enabled it to then be picked up by the Omniprobe, relocated and oriented over the AFM tip prepared for mounting, manipulated into the hole, and finally secured with Pt (Supplementary Fig. 4c). FIB milling at high voltage $(30 \mathrm{kV})$ with $\mathrm{Ga}$ ions was used to remove any excess Pt present on the top and side surfaces and to sharpen the $\mathrm{ZnO} \mathrm{NW}$ into a needle-like shape (Supplementary Fig. 4d). Once prepared, the likelihood of Pt and Ga contamination ${ }^{51}$ was mitigated first by FIB milling at low voltage $(2 \mathrm{kV})$, to reduce the thickness of the damaged zone, and then by polishing the $\mathrm{ZnO} N W$ tip by high-speed scanning in the $\mathrm{AFM}^{54}$ (Supplementary Fig. 4e). $\mathrm{SrTiO}_{3}$ with a high density of nanoparticles was used as the polishing substrate; the height and the size of the nanoparticles were less than 8 and $50 \mathrm{~nm}$, respectively. The resulting $\mathrm{ZnO}$ (0001) AFM tips were atomically flat and oriented, which was confirmed by both SEM and reverse imaging AFM (Fig. 1). EDX analysis detected only Zn and O on the tip surface (inset in Fig. 1b). This fabrication method yielded a series of $\mathrm{ZnO}$ NW tips exposing systematically varying nanoscale areas of the (0001) face so that the contact area dependence of the interfacial force could be determined.

The effective miscut angle of the $\mathrm{ZnO}$ substrate as given by the supplier was $\sim 0.2$ degrees. The effective miscut of the $\mathrm{ZnO}$ tip, on the basis of the step density observed in reverse AFM imaging, was also approximately 0.2 degrees. These are as good as practically achievable on this material.

Analysis of DFS data. The DFS data was analyzed using the generalized multiple bonds model proposed by Friddle, Noy, and De Yoreo ${ }^{29}$. This model provides a comprehensive description of force spectra for a diverse suite of bonds over experimentally relevant pulling rates. The interaction force between two crystal faces is given by:

$$
\begin{gathered}
\langle f\rangle_{N}=f_{\mathrm{eq}}+N f_{\beta} e^{\frac{N}{R\left(\operatorname{leq}_{\mathrm{eq}} / N\right)}} E_{1}\left[\frac{N}{R\left(f_{\mathrm{eq}} / N\right)}\right] \\
R\left(f_{\mathrm{eq}} / N\right)=\frac{r}{k_{\mathrm{u}}\left(f_{\mathrm{eq}} / N\right) f_{\beta}}
\end{gathered}
$$

where $f_{e q}$ is the equilibrium force for the bond/transducer system, $r$ is the loading rate, $\mathrm{N}$ is the equilibrated number of formed bonds at zero force, $f_{\beta}=\frac{k_{B} T}{x_{i}}$ is the thermal force scale, $k_{B}$ is Boltzmann's constant, $T$ is the temperature, $x_{t} x_{t}$ is the barrier location, $E_{1}(z)=\int_{z}^{\infty} \frac{e^{-s}}{s} \mathrm{~d} s$ is the exponential integral, $r$ is the pulling rate, $k_{u}(f)=k_{u}^{0} e^{\beta\left(x_{t}-0.5 k_{c} x_{t}^{2}\right)}$ is the unbinding transition rate, $k_{c}$ is the spring constant of the AFM cantilever and the $k_{u}^{0}$ is the intrinsic unbinding rate.

The resulting equilibrium interaction forces for different azimuthal orientation were fit using the sinusoidal waveform:

$$
f_{\text {eq }}=f_{0}+A \times \sin \left(2 \pi \frac{x-x c}{\omega}\right)
$$

where $f_{0}$ is the offset, $A$ is the amplitude, $x$ is the azimuthal orientation, $x c$ is the phase shift, and the $\omega$ is the period.

Classical MD simulations. Classical MD simulations were performed at room temperature and zero-applied pressure by using DL_POLY Classic ${ }^{55}$. The calculations were conducted in the NVT ensemble using the Nosé-Hoover thermostat ${ }^{56}$. The equations of motion were integrated using the Verlet-leapfrog algorithm with a time step of 2 fs. The Ewald sum method was used to calculate the long-range Coulombic interactions with a real-space cutoff of $15 \AA$. The same cutoff distance was used for the short-range interactions. The force fields used in this study are described in the Supplementary Table 1. The $\mathrm{ZnO}$ substrate had dimensions of approximately $59 \times 51 \times 24 \AA^{3}$ and it consisted of $10 \mathrm{Zn}$-O double layers, with the middle four layers fixed. $25 \%$ of the surface $\mathrm{Zn}$ or $\mathrm{O}$ atoms on the respective surface of the $\mathrm{ZnO}$ substrate were removed in order to eliminate the net dipole normal to the (0001) surfaces. The $\mathrm{ZnO}$ nanoparticle consisted of seven $\mathrm{Zn}$-O double layers and a (0001) surface area of about $340 \AA^{2}$. No vacancies were introduced on the $(0001)$ and $(000 \overline{1})$ surfaces of the $\mathrm{ZnO}$ particle as zero net dipole was predicted by METADISE ${ }^{57}$. Steps on basal surfaces of both the nanoparticle and the substrate were not included in our simulations since there was no obvious four-fold symmetry in the rupture force data and thus the step perturbation is trivial. The water slab had a density of $\sim 0.96 \mathrm{~g} / \mathrm{cm}^{3}$. In order to keep the surface $\mathrm{Zn}$ atoms of the bottom substrate from dissolving into the solution, a harmonic tethering potential was applied to those $\mathrm{Zn}$ atoms (Supplementary Table 1). In PMF calculations (Supplementary Note 5), the orientation of the substrate was kept constant while the nanoparticle was initially rotated from $0^{\circ}$ to $120^{\circ}$ with an interval of $15^{\circ}$. At each angle, a system with two surfaces separated by two water layers in solution was run for 2 ns (Supplementary Movie 3), whose output was used for the starting structure for further PMF calculations in respective orientation case. In each PMF calculation, the system was first equilibrated for $200 \mathrm{ps}$ and then was run for $3 \mathrm{~ns}$ for production. The force to constrain the particle along the normal to the basal plane was collected for the last $1.2 \mathrm{~ns}$. In cases where the force was not converged within the first $3 \mathrm{~ns}$, the simulations were extended for another $2 \mathrm{~ns}$ to ensure convergence. The hydroxylation effect on the surface water structure was assessed in Supplementary Note 6 and Supplementary Fig. 12.

Data availability. Data supporting the conclusions presented in this study are available from corresponding author upon request.

Received: 10 January 2017 Accepted: 28 July 2017

Published online: 10 October 2017

\section{References}

1. De Yoreo, J. J. et al. Crystallization by particle attachment in synthetic, biogenic, and geologic environments. Science 349, aaa6760 (2015).

2. Banfield, J. F., Welch, S. A., Zhang, H., Ebert, T. T. \& Penn, R. L. Aggregationbased crystal growth and microstructure development in natural iron oxyhydroxide biomineralization products. Science 289, 751-754 (2000).

3. Penn, R. L. \& Banfield, J. F. Imperfect oriented attachment: dislocation generation in defect-free nanocrystals. Science 281, 969-971 (1998).

4. Yang, M. et al. Self-assembly of nanoparticles into biomimetic capsid-like nanoshells. Nat. Chem. 9, 287-294 (2016).

5. Weidman, M. C., Smilgies, D. M. \& Tisdale, W. A. Kinetics of the self-assembly of nanocrystal superlattices measured by real-time in situ X-ray scattering. Nat. Mater. 15, 775-781 (2016).

6. Kundu, P. K. et al. Light-controlled self-assembly of non-photoresponsive nanoparticles. Nat. Chem. 7, 646-652 (2015).

7. Halder, A. \& Ravishankar, N. Ultrafine single-crystalline gold nanowire arrays by oriented attachment. Adv. Mater. 19, 1854-1858 (2007).

8. Liu, Z. et al. Synthesis of silver nanoplates by two-dimensional oriented attachment. Langmuir 28, 9244-9249 (2012).

9. Yong, K. T. et al. Formation of ZnTe nanowires by oriented attachment. Chem. Mater. 19, 4108-4110 (2007).

10. Yu, X., Wang, D., Peng, Q. \& Li, Y. Pt-M $(\mathrm{M}=\mathrm{Cu}, \mathrm{Co}, \mathrm{Ni}, \mathrm{Fe})$ nanocrystals: from small nanoparticles to wormlike nanowires by oriented attachment. Chem. Eur. J. 19, 233-239 (2013).

11. Liao, H.-G., Cui, L., Whitelam, S. \& Zheng, H. Real-time imaging of $\mathrm{Pt}_{3} \mathrm{Fe}$ nanorod growth in solution. Science 336, 1011-1014 (2012).

12. Pacholski, C., Kornowski, A. \& Weller, H. Self-assembly of ZnO: from nanodots to nanorods. Angew. Chem. Int. Ed. 41, 1188-1191 (2002).

13. Fan, B., Zhang, Y., Yan, R. \& Fan, J. Multistage growth of monocrystalline $\mathrm{ZnO}$ nanowires and twin-nanorods: oriented attachment and role of the spontaneous polarization force. CrystEngComm. 18, 6492-6501 (2016).

14. Zitoun, D., Pinna, N., Frolet, N. \& Belin, C. Single crystal manganese oxide multipods by oriented attachment. J. Am. Chem. Soc. 127, 15034-15035 (2005)

15. Chen, J. S., Zhu, T., Li, C. M. \& Lou, X. W. Building hematite nanostructures by oriented attachment. Angew Chem. Int. Ed. 50, 650-653 (2011).

16. Schliehe, C. et al. Ultrathin PbS sheets by two-dimensional oriented attachment. Science 329, 550-553 (2010).

17. Zhang, J. et al. A multistep oriented attachment kinetics: coarsening of $\mathrm{ZnS}$ nanoparticle in concentrated NaOH. J. Am. Chem. Soc. 128, 12981-12987 (2006).

18. O'Sullivan, C. et al. Spontaneous room temperature elongation of $\mathrm{CdS}$ and $\mathrm{Ag}_{2} \mathrm{~S}$ nanorods via oriented attachment. J. Am. Chem. Soc. 131, 12250-12257 (2009).

19. Pradhan, N., Xu, H. \& Peng, X. Colloidal CdSe quantum wires by oriented attachment. Nano Lett. 6, 720-724 (2006).

20. Cho, K. S., Talapin, D. V., Gaschler, W. \& Murray, C. B. Designing PbSe nanowires and nanorings through oriented attachment of nanoparticles. J. Am. Chem. Soc. 127, 7140-7147 (2005).

21. Du, W., Qian, X., Niu, X. \& Gong, Q. Symmetrical six-horn nickel diselenide nanostars growth from oriented attachment mechanism. Cryst. Growth Des. 7 2733-2737 (2007).

22. Colfen, H. \& Antonietti, M. Mesocrystals: inorganic superstructures made by highly parallel crystallization and controlled alignment. Angew. Chem. Int. Ed. Engl. 44, 5576-5591 (2005) 
23. Javon, E. et al. Competing forces in the self-assembly of coupled $\mathrm{ZnO}$ nanopyramids. ACS nano 9, 3685-3694 (2015).

24. Song, R. Q. \& Colfen, H. Mesocrystals-ordered nanoparticle superstructures. Adv. Mater. 22, 1301-1330 (2010).

25. Li, D. et al. Direction-specific interactions control crystal growth by oriented attachment. Science 336, 1014-1018 (2012).

26. McGuiggan, P. M. \& Israelachvili, J. N. Adhesion and short-range forces between surfaces (II). Part II: Effects of surface lattice mismatch. J. Mater. Res. 5, 2232-2243 (1990).

27. Finot, E., Lesniewska, E., Mutin, J.-C. \& Goudonnet, J.-P. Investigations of surface forces between gypsum microcrystals in air using atomic force microscopy. Langmuir. 16, 4237-4244 (2000).

28. Zhang, X. et al. Direction-specific van der Waals attraction between rutile $\mathrm{TiO}_{2}$ nanocrystals. Science 365, 434-437 (2017).

29. Friddle, R. W., Aleksandr, N. \& De Yoreo, J. J. Interpreting the widespread nonlinear force spectra of intermolecular bonds. Proc. Natl Acad. Sci. USA 109, 13573-13578 (2012).

30. Wang, Z. L. Zinc oxide nanostructures: growth, properties and applications. J. Phys. Condens. Matter. 16, R829-R858 (2004).

31. Wang, Z. L. Nanostructures of zinc oxide. Mater. Today 7, 26-33 (2004).

32. Tian, Z. R. et al. Complex and oriented $\mathrm{ZnO}$ nanostructures. Nat. Mater. 2, 821-826 (2003).

33. Wang, Z. L. \& Song, J. Piezoelectric nanogenerators based on zinc oxide nanowire arrays. Science 312, 242-246 (2006).

34. Ko, S. H. et al. Nanoforest of hydrothermally grown hierarchical $\mathrm{ZnO}$ nanowires for a high efficiency dye-sensitized solar cell. Nano Lett. 11, 666-671 (2011).

35. Repins, I. et al. $19 \cdot 9 \%$-efficient $\mathrm{ZnO} / \mathrm{CdS} / \mathrm{CuInGaSe}_{2}$ solar cell with $81 \cdot 2 \%$ fill factor. Prog. Photovoltaics 16, 235-239 (2008).

36. Hwang, D.-K. et al. p- $\mathrm{ZnO} / \mathrm{n}-\mathrm{GaN}$ heterostructure $\mathrm{ZnO}$ light-emitting diodes. Appl. Phys. Lett. 86, 222101 (2005).

37. Su, Y. K. et al. Ultraviolet $\mathrm{ZnO}$ nanorod photosensors. Langmuir 26, 603-606 (2010).

38. Behnajady, M. A., Modirshahla, N. \& Hamzavi, R. Kinetic study on photocatalytic degradation of C.I. Acid Yellow 23 by $\mathrm{ZnO}$ photocatalyst. J. Hazard. Mater. 133, 226-232 (2006).

39. Zhang, H. \& Banfield, J. F. Energy calculations predict nanoparticle attachment orientations and asymmetric crystal formation. J. Phys. Chem. Lett. 3, 2882-2886 (2012)

40. Butt, H.-J., Cappella, B. \& Kappl, M. Force measurements with the atomic force microscope: technique, interpretation and applications. Surf. Sci. Rep. 59, 1-152 (2005).

41. Wander, A. et al. Stability of polar oxide surfaces. Phys. Rev. Lett. 86, 3811-3814 (2001).

42. Zhang, P. et al. Surface enthalpies of nanophase $\mathrm{ZnO}$ with different morphologies. Chem. Mater. 19, 5687-5693 (2007).

43. Stampfl, C. \& Van de Walle, C. G. Energetics and electronic structure of stacking faults in AlN, GaN, and InN. Phys. Rev. B 57, R15052 (1998).

44. Yan, Y., Dalpian, G. M., Al-Jassim, M. M. \& Wei, S. H. Energetics and electronic structure of stacking faults in ZnO. Phys. Rev. B 70, 193206 (2004).

45. Israelachvili, J. N. Adhesion forces between surfaces in liquids and condensable vapours. Surf. Sci. Rep. 14, 109-159 (1992).

46. Schneck, E., Sedlmeier, F. \& Netz, R. R. Hydration repulsion between biomembranes results from an interplay of dehydration and depolarization. Proc. Natl Acad. Sci. USA 109, 14405-14409 (2012).

47. Kanduč, M., Schneck, E. \& Netz, R. R. Attraction between hydrated hydrophilic surfaces. Chem. Phys. Lett. 610, 375-380 (2014).

48. Ong, Q. K. \& Sokolov, I. Attachment of nanoparticles to the AFM tips for direct measurements of interaction between a single nanoparticle and surfaces. $J$. Colloid Interface Sci. 310, 385-390 (2007)

49. Kinoshita, Y., Naitoh, Y., Li, Y. J. \& Sugawara, Y. Fabrication of sharp tungstencoated tip for atomic force microscopy by ion-beam sputter deposition. Rev. Sci. Instrum. 82, 113707 (2011).

50. Lugstein, A., Bertagnolli, E., Kranz, C. \& Mizaikoff, B. Fabrication of a ring nanoelectrode in an AFM tip: novel approach towards simultaneous electrochemical and topographical imaging. Surf. Interface Anal. 33, 146-150 (2002).

51. Thompson, K. et al. In situ site-specific specimen preparation for atom probe tomography. Ultramicroscopy 107, 131-139 (2007).
52. $\mathrm{Xu}, \mathrm{S}$. et al. Optimizing and improving the growth quality of $\mathrm{ZnO}$ nanowire arrays guided by statistical design of experiments. ACS Nano 3, 1803-1812 (2009).

53. Wang, Z. L. ZnO nanowire and nanobelt platform for nanotechnology. Mater. Sci. Eng. R 64, 33-71 (2009).

54. Marchetto, D. et al. AFM investigation of tribological properties of nanopatterned silicon surface. Wear 265, 577-582 (2008).

55. Smith, W. \& Forester, T. R. DL_POLY_2.0: A general-purpose parallel molecular dynamics simulation package. J. Mol. Graph. 14, 136-141 (1996).

56. Hoover, W. G. Canonical dynamics: equilibrium phase-space distributions. Phys. Rev. A 31, 1695 (1985).

57. Watson, G. W., Kelsey, E. T., de Leeuw, N. H., Harris, D. J. \& Parker, S. C. Atomistic simulation of dislocations, surfaces and interfaces in $\mathrm{MgO}$. J. Chem Soc. Faraday Trans. 92, 433-438 (1996).

\section{Acknowledgements}

This material is based upon work supported by the US Department of Energy (DOE), Office of Science, Office of Basic Energy Sciences, Chemical Sciences, Geosciences, and Biosciences Division through its Geosciences program at Pacific Northwest National Laboratory (PNNL). The Materials Synthesis and Simulation Across Scales Initiative, a Laboratory Directed Research and Development program at PNNL, supported development of tip fabrication methods and the large-scale MD methods. The authors acknowledge fruitful discussions with C.J. Mundy on the computer simulations and theoretical analysis, and with J. Tao on the dynamic force measurements. Tip fabrication and characterization were performed using the Environmental and Molecular Sciences Laboratory (EMSL), a national scientific user facility at PNNL sponsored by the DOE's Office of Biological and Environmental Research. PNNL is a multi-program national laboratory operated by Battelle Memorial Institute under Contract No. DE-AC0576RL01830 for the US Department of Energy.

\section{Author contributions}

X.Z., J.J.D.Y. and K.M.R. conceived and designed the experiments. X.Z. and J.L. developed the crystal AFM tip fabrication method and fabricated all tips. M.E.B. conducted the XRD analyses. X.Z. conducted the AFM experiments and force data analysis. Z.S., S.N.K., M.L.S., and K.M.R. performed the computer simulations and theoretical analysis. X.Z., Z.S., and K.M.R. co-wrote the paper. All authors discussed the results and commented on the manuscript.

\section{Additional information}

Supplementary Information accompanies this paper at doi:10.1038/s41467-017-00844-6.

Competing interests: The authors declare no competing financial interests.

Reprints and permission information is available online at http://npg.nature.com/ reprintsandpermissions/

Publisher's note: Springer Nature remains neutral with regard to jurisdictional claims in published maps and institutional affiliations.

Open Access This article is licensed under a Creative Common Attribution 4.0 International License, which permits use, sharing, adaptation, distribution and reproduction in any medium or format, as long as you give appropriate credit to the original author(s) and the source, provide a link to the Creative Commons license, and indicate if changes were made. The images or other third party material in this article are included in the article's Creative Commons license, unless indicated otherwise in a credit line to the material. If material is not included in the article's Creative Commons license and your intended use is not permitted by statutory regulation or exceeds the permitted use, you will need to obtain permission directly from the copyright holder. To view a copy of this license, visit http://creativecommons.org/ licenses/by/4.0/.

(C) The Author(s) 2017 